\title{
A single full gap with mixed type-I and type-II superconductivity on surface of the type-II Dirac semimetal PdTe 2 by point-contact spectroscopy
}

\author{
Tian Le, ${ }^{1}$ Lichang Yin, ${ }^{1}$ Zili Feng, ${ }^{2}$ Qi Huang, ${ }^{1}$ Liqiang Che, ${ }^{1}$ Jie Li, ${ }^{1}$ Youguo Shi, ${ }^{2,3}$ and Xin $\mathrm{Lu}^{1,4,5, *}$ \\ ${ }^{1}$ Center for Correlated Matter and Department of Physics, Zhejiang University, Hangzhou 310058, China \\ ${ }^{2}$ Beijing National Laboratory for Condensed Matter Physics, \\ Institute of Physics, Chinese Academy of Sciences, Beijing 100190, China \\ ${ }^{3}$ School of Physical Sciences, University of Chinese Academy of Sciences, Beijing 100049, China \\ ${ }^{4}$ Zhejiang Province Key Laboratory of Quantum Technology and Device, Zhejiang University, Hangzhou 310027, China \\ ${ }^{5}$ Collaborative Innovation Center of Advanced Microstructures, Nanjing University, Nanjing, 210093, China
}

(Dated: June 4, 2019)

\begin{abstract}
We report our point-contact spectroscopy (PCS) study on the superconducting state of the type-II Dirac semimetal $\mathrm{PdTe}_{2}$ with a superconducting transition temperature $T_{\mathrm{c}} \sim 1.65 \mathrm{~K}$. Both mechanical- and soft- PCS differential conductance curves at $0.3 \mathrm{~K}$ show a consistent double-peak structure and they can be perfectly fitted by a single s-wave gap based on the Blonder-TinkhamKlapwijk model. The gap follows a typical Bardeen-Cooper-Schrieffer temperature behavior, yielding $\Delta_{0} \sim 0.29 \mathrm{meV}$ and $2 \Delta_{0} / k_{\mathrm{B}} T_{\mathrm{c}}=4.15$ in the strong coupling regime. A sudden suppression of the superconducting gap in magnetic field around $H_{\mathrm{c} 1} \sim 130$ Oe is observed for most point-contacts on $\mathrm{PdTe}_{2}$, characteristic of a first-order transition for type-I superconductor in field. However, for other contacts, a smooth evolution of the PCS conductance persists up to $H_{\mathrm{c} 2} \sim 600$ Oe, signaling a local type-II superconductivity. The observed admixture of type-I and type-II superconductivity can possibly arise from an inhomogeneous electron mean free path on the surface of $\mathrm{PdTe}_{2}$ due to its topological surface states.
\end{abstract}

Soon after the discovery of topological insulators (TIs), topological superconductors (TSCs) have also attracted intensive attention in the community because of the nontrivial topology for Bogoliubov quasiparticles in TSCs and electronic bands in TIs [1-8]. Moreover, Majorana fermions are expected to be hosted on the surface or edge of TSCs, complying with non-Abelian statistics and playing a decisive role in quantum computation [9-11]. One strategy to realize TSCs is to induce superconductivity (SC) in topological materials, such as topological insulator, Weyl and Dirac semimetals, by taking advantage of the nontrivial topology of the electronic bands [1218]. For example, topological SC has been claimed for the topological surface states with proximity-induced SC at the interface of a TI/SC heterostructure or on the surface of some iron-based superconductors, where Majorana zero modes are argued to exist at their vortices or edges [19-28].

Recently, the transition-metal dichalcogenide compound $\mathrm{PdTe}_{2}$ has been confirmed to be a type-II Dirac semimetal with a tilted Dirac cone below the Fermi energy by the angle resolved photoemission spectroscopy (ARPES) and there exists a spin-polarized topological surface state $[29,30]$. De Haas-van Alphen (dHvA) experiments also evidence the nontrivial Berry phase for one of the Fermi-surface pockets, probably a hole pocket from the tilted Dirac cone [31, 32]. On the other hand, superconductivity in $\mathrm{PdTe}_{2}$ with a transition temperature $T_{\mathrm{c}} \sim 1.7 \mathrm{~K}$ has been known for a long time, serving as a promising candidate for stoichiometric TSCs [33]. However, heat capacity, London penetration depth, tunneling junction and STM measurements all support a con- ventional fully-gapped s-wave superconductor for $\mathrm{PdTe}_{2}$ [30, 34-38]. Moreover, recent electrical transport and heat capacity measurements reveal a puzzling discrepancy of its critical field for the superconducting state with 3000 Oe and 250 Oe, respectively. It is difficult to be explained by either a general Saint-James-de Gennes (SJdG) surface critical field or filamentary superconductivity [33, 34]. One possible scenario proposed by Siroshi et al. is that bulk $\mathrm{PdTe}_{2}$ is a typical type-I superconductor and there exists a mixing of type-I and type-II superconductivity on its surface [33, 34,37]. More experiments are thus desired to elucidate its exact superconducting nature for the type-II Dirac semimetal $\mathrm{PdTe}_{2}$.

In this article, we have applied both mechanical and soft point-contact spectroscopy (PCS) to investigate the superconducting gap of single crystalline $\mathrm{PdTe}_{2}$. Our PCS conductance curves at $0.3 \mathrm{~K}$ can be well fitted by a single gap s-wave Blonder-Tinkham-Klapwijk (BTK) model [39] and the temperature evolution of the extracted superconducting gap follows a conventional Bardeen-Cooper-Schrieffer (BCS) behavior, yielding a gap $\Delta_{0} \sim 0.29 \mathrm{meV}$ with $2 \Delta_{0} / k_{\mathrm{B}} T_{\mathrm{c}}=4.15$. Even though some point-contacts show an abrupt suppression of the SC gap by magnetic field at 130 Oe, characteristic of a type-I superconductor, some other contacts exhibit a smooth evolution in field with a typical type-II superconducting behavior below a critical field of 600 Oe, suggesting an inhomogeneous mixing of type-I and type-II SC on the $\mathrm{PdTe}_{2}$ crystal surface.

$\mathrm{PdTe}_{2}$ single crystals were grown by the flux method: High purity $\mathrm{Pd}$ and Te with the molar ratio 1:4 were put into an aluminum crucible and then sealed in a quartz 

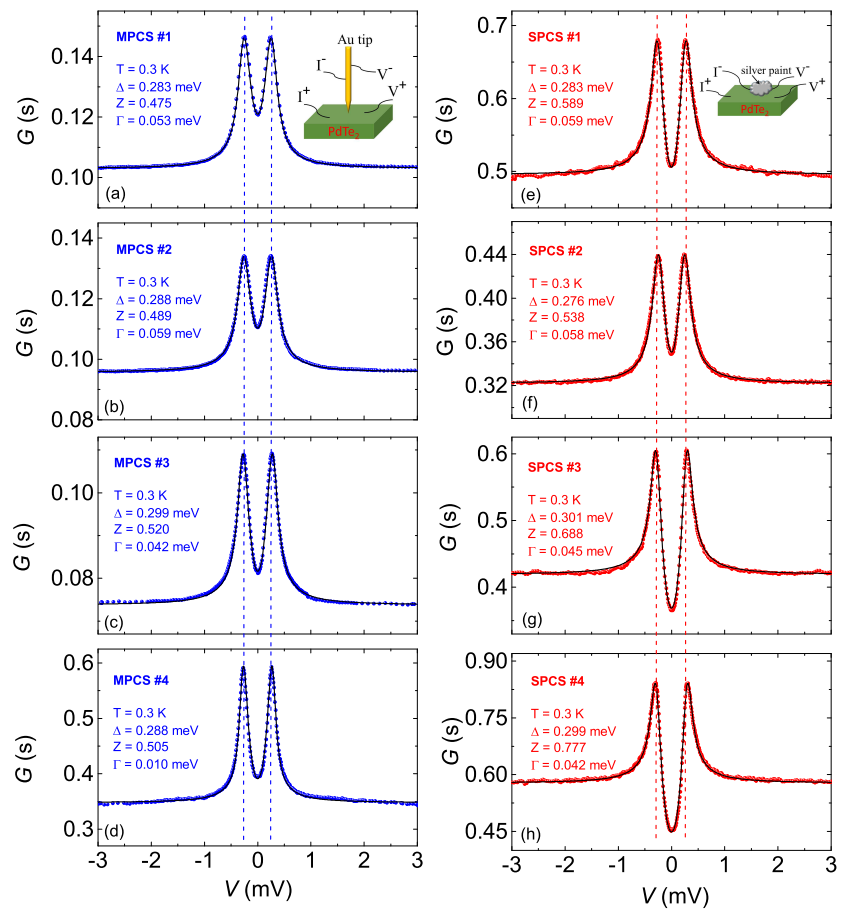

FIG. 1. (color online) A representative set of point-contact conductance curves on $\mathrm{PdTe}_{2}$ at $0.3 \mathrm{~K}$ for different contacts from MPCS (a-d) and SPCS (e-h) in comparison with their optimized single gap s-wave BTK fittings (black lines). The insets in (a) and (e) are schematic illustrations for MPCS and SPCS, respectively.

tube. The sample was heated up to $800{ }^{\circ} \mathrm{C}$ and then cooled to $500{ }^{\circ} \mathrm{C}$ slowly. The excess Te were removed by centrifuge at this temperature and single crystals $\mathrm{PdTe}_{2}$ were left at the bottom of the crucible. Before experiment, the samples were cleaved to expose fresh surface at ambient condition. Mechanical PCS (MPCS) in a needle-anvil configuration was employed, where an electrochemically-etched sharp gold tip was gently engaged on the crystal surface by piezo-controlled nanopositioners. In comparison, soft PCS (SPCS) contacts on $\mathrm{PdTe}_{2}$ were prepared on the sample surface by attaching a $30 \mu \mathrm{m}$ diam gold wire with a silver-paint drop at the end yielding a total contact diameter around $\sim$ 50-100 $\mu \mathrm{m}$. Hundreds of parallel nanoscale junctions are assumed between individual silver particles and the crystal surface for SPCS [40, 41]. In the case of MPCS, even though parallel nanoscale channels should also exist, the total contact diameter from the sharp tip end is less than $5 \mu \mathrm{m}$, much smaller than that of SPCS. The PCS differential conductance curves as a function of bias voltage, $G(V)$, were recorded with the conventional lock-in technique in a quasi-four-probe configuration. Oxford cryostat with a He3 insert was used to cool the whole PCS setup down to $0.3 \mathrm{~K}$ and magnetic field was applied along the crystal c-axis up to 1000 Oe to suppress SC.

Figure 1 shows eight representative PCS differential
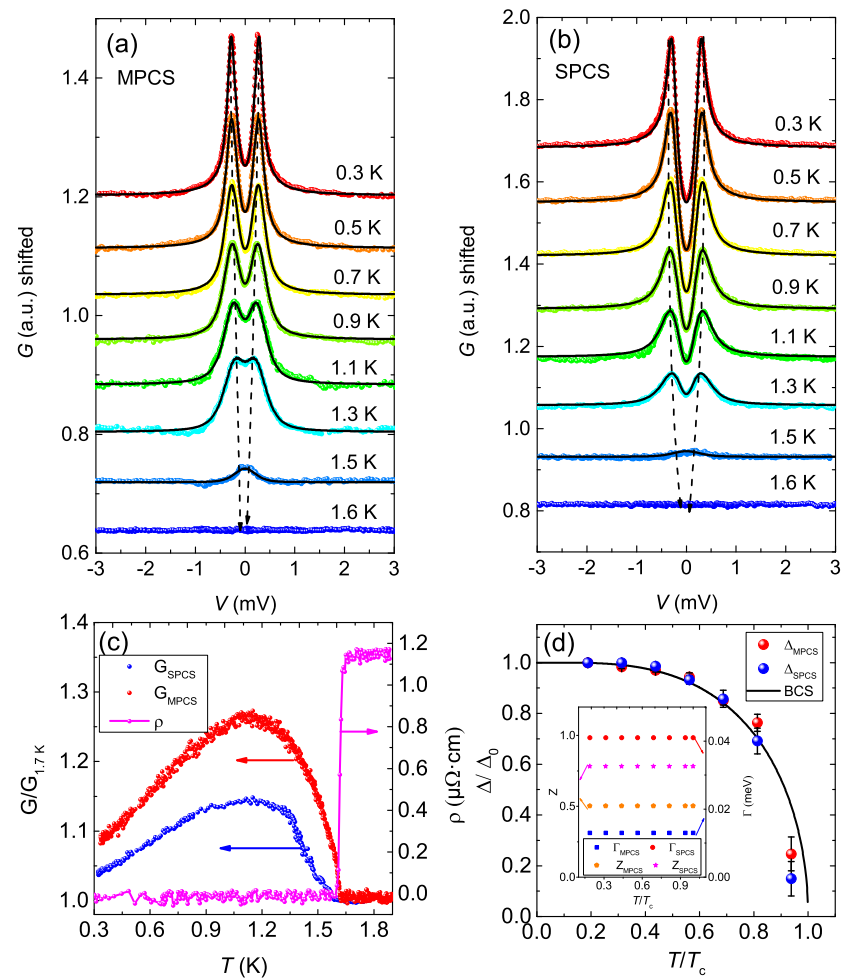

FIG. 2. (color online) (a) and (b) Temperature evolution of the differential conductance curves $G(V)$ from $0.3 \mathrm{~K}$ to 1.6 $\mathrm{K}$ for MPCS and SPCS on $\mathrm{PdTe}_{2}$, respectively, in comparison with a single gap s-wave BTK fitting (black lines). (c) Temperature dependence of the electrical resistivity $\rho$ (pink dots) and normalized zero-bias conductance $G / G_{1.7 K}$ curves from MPCS (red dots) and SPCS (blue dots), respectively. (d) Temperature dependence of the extracted superconducting gap $\Delta$ from the single-gap BTK fitting for both MPCS and SPCS in accordance with the BCS curve. The inset shows little change of fitting parameters $\mathrm{Z}$ and $\Gamma$ in MPCS and SPCS as a function of temperature.

conductance curves $G(V)$ on $\mathrm{PdTe}_{2}$ at the lowest temperature $0.3 \mathrm{~K}$ with the left and right panel for MPCS and SPCS, respectively. All the conductance curves show a common double-peak structure around $\pm 0.3 \mathrm{meV}$ and no dip feature at high bias is present in any curve, ensuring the ballistic nature of our contacts [42]. A single gap swave BTK model can perfectly fit all experimental curves as shown by the black lines in Fig. 1 and the extracted superconducting gap $\Delta$ values at $0.3 \mathrm{~K}$ are scattered in the range of 0.276 and $0.301 \mathrm{meV}$, yielding $2 \Delta / k_{\mathrm{B}} T_{\mathrm{c}}=$ $4.00-4.36$ in a strong-coupling regime. We notice that our PCS gap values are consistent with those reported by STM $(\sim 0.289 \mathrm{meV})[37,38]$, but larger than the weakcoupling value estimated from bulk specific heat and penetration depth studies [34, 35]. The discrepancy of gap size is suspected to be associated with the spin-polarized topological surface state, because both PCS and STM are more sensitive to the SC on surface rather than the bulk SC. However, its exact origin needs further clarifica- 

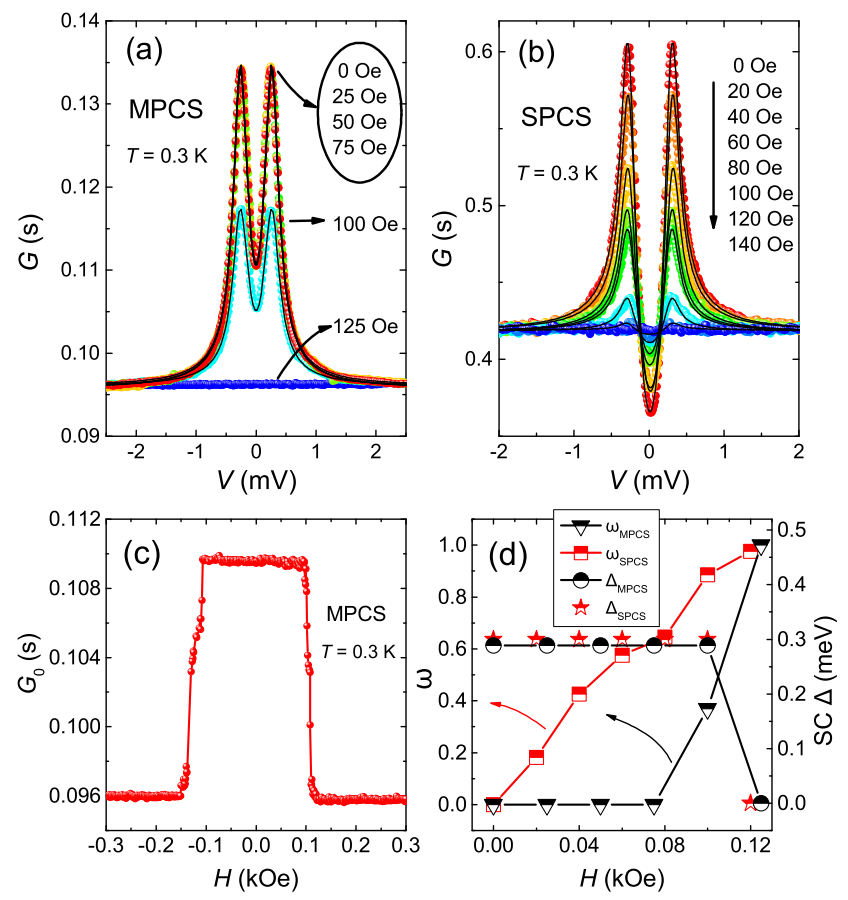

FIG. 3. (color online) (a) and (b) Point-contact differential conductance curves $G(V)$ at $0.3 \mathrm{~K}$ as a function of magnetic field for MPCS and SPCS on $\mathrm{PdTe}_{2}$, respectively, in comparison with a two-component BTK fitting (black lines). (c) Field dependence of the MPCS zero-bias conductance has a clear first-order transition and the residual magnetic field is estimated 15 Oe. (d) Field evolution of the extracted spectra weight $\omega$ for normal state and the superconducting gap $\Delta$ for $\mathrm{SC}$ regions for both MPCS and SPCS.

tion [43-45]. For optimal fittings, the smearing parameter $\Gamma$ is much smaller than the superconducting gap and comparable between MPCS and SPCS, while the barrier strength parameter $\mathrm{Z}$ ranges from $\sim 0.5$ to 0.78 for different contacts in Fig. 1 possibly from the Fermi velocity mismatch between the tip and sample [39, 46].

Figure 2(a) and (b) show the temperature evolution of conductance curves $G(V)$ from 0.3 to $1.6 \mathrm{~K}$ for MPCS and SPCS on $\mathrm{PdTe}_{2}$, respectively. With increased temperatures, the double peaks are smeared to a single peak and finally disappear at the superconducting $T_{\mathrm{c}} \sim 1.65$ $\mathrm{K}$ for $\mathrm{PdTe}_{2}$. We note that the $T_{\mathrm{c}}$ determined by the zero-bias conductance (ZBC) curves for both mechanical and soft contacts are around $1.65 \mathrm{~K}$, consistent with the resistive $T_{\mathrm{c}}$ as shown in Fig. 2(c). The extracted $\mathrm{SC}$ gap values from an optimal BTK fitting are shown in Fig. 2(d) and it follows the conventional BCS temperature behavior. As in the inset of Fig. 2(d), the fitting parameters $\mathrm{Z}$ and $\Gamma$ for both MPCS and SPCS show little change with temperature, indicating an ideal ballistic contact [47].

For the MPCS field dependence, the conductance curves $G(V)$ show an interesting behavior as in Fig. 3(a): They barely change but overlap with each other below 75
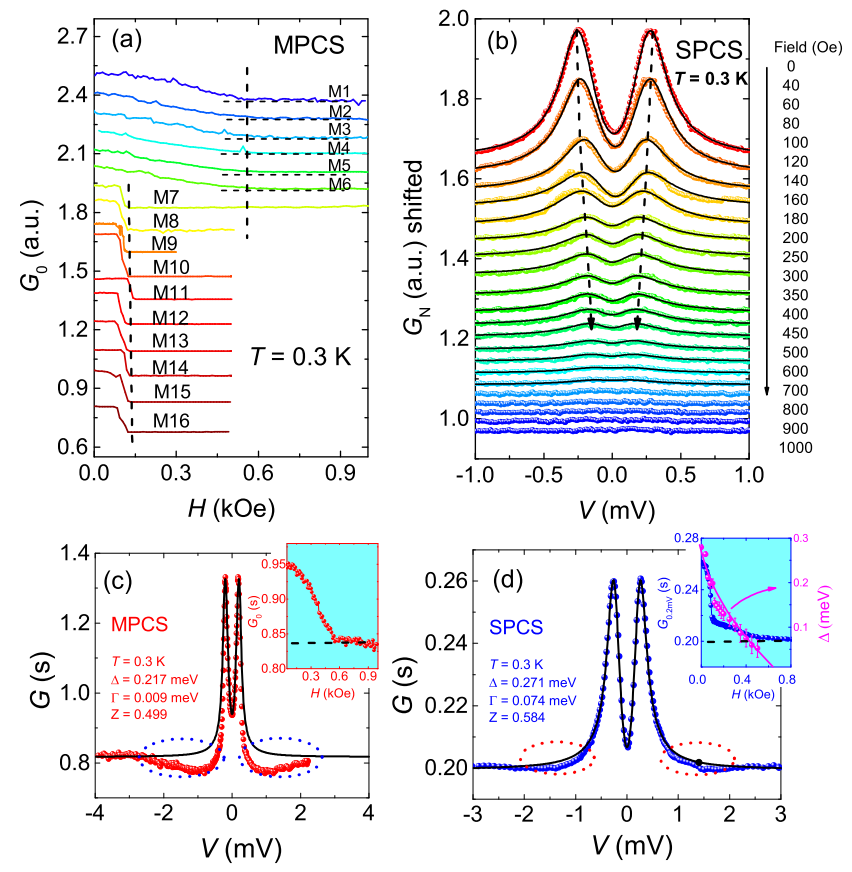

FIG. 4. (color online) (a) Field dependence of the zero-bias conductance for different MPCS contacts on the surface of $\mathrm{PdTe}_{2}$. (b) One set of SPCS conductance curves as a function of field show a type-II SC behavior at $0.3 \mathrm{~K}$ in comparison with the standard BTK fitting (black lines). (c)-(d) The typical conductance curve $G(V)$ for a type-II SC behavior at 0.3 $\mathrm{K}$ has a dip structure at high bias for MPCS and SPCS, respectively. The insets show the field-dependent contact conductance at zero-bias for MPCS and fixed bias voltage 0.2 meV for SPCS, respectively, while the extracted SC gap from the standard BTK fitting has a smooth evolution with field.

Oe, and the conductance peaks are abruptly suppressed in intensity at 100 Oe and quickly disappear around 125 Oe, while the peak positions stay almost unshifted in field. The MPCS zero-bias conductance as a function of field displays a sudden drop at 130 Oe as in Fig. 3(c), strongly supporting a type-I superconductor for $\mathrm{PdTe}_{2}$ as reported by magnetization, heat capacity and STM measurements [33, 34, 37]. In contrast, the SPCS conductance curves for $\mathrm{PdTe}_{2}$ as in Fig. 3(b) show a gradually reduced peak intensity in field, whereas the peak positions have no change before the suppression of SC at 130 Oe. We note that, for type-I superconductors, an intermediate state will emerge far below its critical field when the field is perpendicular to the sample plane with a large demagnetization factor. In such a case, normal state domains can coexist with the SC domains in space and progressively replace the superconducting volume [48-50]. In order to mimic this process, a modified two-component BTK model has been applied to fit the conductance curves with both contributions from the normal and SC regions, $G(V)=\omega G_{\text {Normal }}+$ (1$\omega) G_{\mathrm{BTK}}(V)$, where $G_{\text {Normal }}$ is just flat for the normal state, $G_{\mathrm{BTK}}(V)$ is the standard BTK curve for the SC 
regions, and the fitting parameter $\omega$ denotes the spectra weight from the normal state. The SPCS conductance curves at different fields can be well fitted by this modified BTK model as shown in Fig. 3(b) and the parameters $\mathrm{Z}$ and $\Gamma$ change little with field as in MPCS. The normal state spectra weight $\omega$ shows a roughly linear increase with field as in Fig. 3(d) and vividly illustrates a monotonic increase of the normal state volume in the intermediate state. Nonetheless, the SC gap for the SC regions keeps constant in field up to $100 \mathrm{Oe}$ for $\mathrm{PdTe}_{2}$, indicative of a type-I superconductor. This modified BTK model can also be applicable to the MPCS case as in Fig. 3(d): A sudden increase of the $\omega$ value from zero implies normal state domains entering the contact area, only when the field gets close to $H_{\mathrm{c} 1}$. The systematic difference between MPCS and SPCS is due to its much smaller total contact area in MPCS so that the tip only probes a single superconducting domain for MPCS in low fields.

For MPCS, we have collected a set of ZBC curves as a function of field at $0.3 \mathrm{~K}, G_{0}(H)$, for contacts M1-M16 as shown in Fig. 4(a). Each contact is obtained once after the tip is withdrawn, moved to another position by xy positioners and gently engaged on the sample with the $\mathrm{z}$ positioner, where M1-M16 are from the same tip but not numerically ordered as the experimental sequence. While type-I SC for $\mathrm{PdTe}_{2}$ can been established by the sudden drop at 130 Oe in ZBC for contacts M7-M16, we notice that other ZBC curves M1-M6 show a smooth evolution into the normal state at a critical field $H_{\mathrm{c} 2}$ around 600 Oe. The continuous transition for M1-M6 with a much larger critical field than the bulk $H_{\mathrm{c} 1} \sim 130$ Oe suggests a local type-II superconductivity at these contacts, consistent with the STM and resistivity measurements for current along c axis [31, 37]. A corresponding conductance curve $G(V)$ at $0.3 \mathrm{~K}$ has an obvious dip structure at higher bias voltages as in Fig. 4(c), implying the contact not in a pure ballistic limit and the mean free path $l$ is believed smaller than the diameter of each contact channel [42]. It is intriguing that no additional dips are observed for any contact among M7- M16 with type-I SC, while dips are commonly observed for contacts M1M6 with type-II SC. Some SPCS conductance curves also exhibit a smooth evolution of the SC gap with field as in Fig. 4(b) and the inset of Fig. 4(d). Its conductance at a fixed bias voltage $0.2 \mathrm{meV}$ shows a similar continuous type-II behavior at 600 Oe after a first-order-like drop at 130 Oe as in the inset of Fig. 4(d), signaling a spacial coexistence of type-I and type-II SC in the soft contact area (We note that the zero-bias conductance for our SPCS contacts available is comparable to the value in the normal state, and so the conductance at a fixed bias voltage $0.2 \mathrm{meV}$ rather rather than zero bias is chosen to easily identify the critical field). A weak dip structure at high bias is also observed with the conductance curve deviating from its BTK fitting as in Fig. 4(d). We notice that

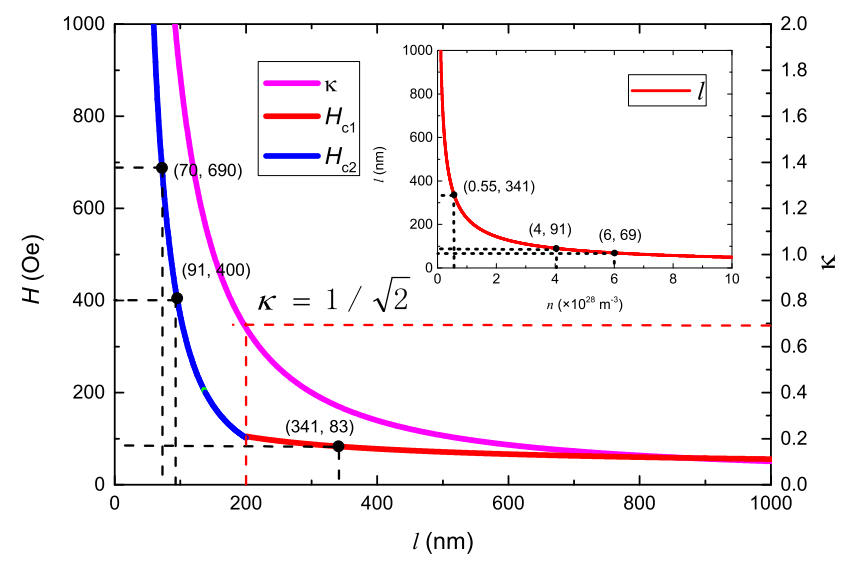

FIG. 5. (color online) Mean free path $l$ dependence of the Ginzburg-Landau parameter $\kappa$ (pink curve) and the corresponding critical fields for type-I and type-II superconductor regimes (red and blue segments, respectively). The inset shows the calculated mean free path $l$ as a function of carry density $n$ based on the Drude model.

a similar contact-dependence for PCS spectra has been reported in a recent study on the non-centrosymmetric superconductor $\mathrm{Re}_{6} \mathrm{Zr}$ and it is ascribed to the surface superconductivity as well [51].

In general, the Ginzburg-Landau (GL) parameter $\kappa=$ $\frac{\lambda}{\xi}$ is defined to describe type-I $\left(\kappa<\frac{1}{\sqrt{2}}\right)$ and type-II $(\kappa>$ $\left.\frac{1}{\sqrt{2}}\right)$ superconductors, where $\lambda$ is the penetration depth and $\xi$ the coherence length of superconductors [52]. It was claimed that the $\kappa$ in $\mathrm{PdTe}_{2}$ is around $0.34<\frac{1}{\sqrt{2}}$, categorizing $\mathrm{PdTe}_{2}$ as a type-I superconductor [33]. In order to explain the emergence of type-II SC behavior, we follow the arguments by Siroshi et al. and consider the Pippard nonlocal electrodynamics $\frac{1}{\xi}=\frac{1}{\xi_{0}}+\frac{1}{l}$ and $\lambda=$ $\lambda_{L} \sqrt{1+\frac{\xi_{0}}{l}}$, where $\xi_{0}$ and $\lambda_{L}$ is the BCS coherence length and London penetration depth with $l \rightarrow \infty$, respectively. Following the relations $\xi_{0}=\frac{0.18 \hbar^{2} k_{F}}{k_{B} T_{c} m^{*}}$ and $\lambda_{L}=\sqrt{\frac{m^{*}}{\mu_{0} n e^{2}}}$, if we assume the carrier density $\mathrm{n}=5.5 \times 10^{27} \mathrm{~m}^{-1}[33]$, the effective mass $m^{*} \approx 0.3 m_{e}[31,53]\left(m_{e}\right.$ is the free electron mass), and the Fermi wave number $k_{F}=\sqrt[3]{3 \pi^{2} n}$ $=5.5 \times 10^{9} \mathrm{~m}^{-1}$, we can get $\lambda_{L}=39 \mathrm{~nm}$ and $\xi_{0}=1800$ $\mathrm{nm}$ for $\mathrm{PdTe}_{2}$. Since $\kappa=\lambda_{L}\left(\frac{1}{\xi_{0}}+\frac{1}{l}\right) \sqrt{1+\frac{\xi_{0}}{l}}, \kappa$ as a function of $l$ is plotted in Fig. 5 as the pink line. We note that the mean free path $l$ for bulk $\mathrm{PdTe}_{2}$ is estimated $l \approx 341 \mathrm{~nm}$ from the Drude model $l=\frac{3 \pi^{2} \hbar}{\rho e^{2} k_{F}^{2}}$, which makes $\kappa \sim 0.341$ in the type-I superconductor regime. A reduced mean free path $l$ on some surface regions can cause an enhanced $\kappa$ and thus tune it into a type-II SC with $l \leq 200 \mathrm{~nm}$. We thus argue that the dip structure at high bias frequently observed for contacts with the type-II SC behavior implies a greatly reduced mean free path $l$, which makes local surface SC regions enter into the type-II regime. 
As for the critical field, according to the GL theory, $H_{\mathrm{c} 1}=\frac{\phi_{0}}{2 \sqrt{2} \pi \mu_{0} \lambda \xi}=\frac{\phi_{0}}{2 \sqrt{2} \pi \mu_{0} \lambda_{L} \sqrt{\xi_{0}}} \sqrt{\frac{1}{\xi_{0}}+\frac{1}{l}}$ and $H_{\mathrm{c} 2}=$ $\frac{\phi_{0}}{2 \pi \mu_{0} \xi^{2}}=\frac{\phi_{0}}{2 \pi \mu_{0}}\left(\frac{1}{\xi_{0}}+\frac{1}{l}\right)^{2}$. The dependence of $H_{\mathrm{c} 1}$ and $H_{\mathrm{c} 2}$ on the mean free path $l$ is shown in Fig. 5 with red and blue segments, respectively. A mean free path $l \sim 341 \mathrm{~nm}$ estimated from the Drude model will give an $H_{\mathrm{c} 1}$ around 83 Oe not so off from our PCS value 130 Oe for type-I SC behavior. As shown in Fig. 5, a slight variation of the mean free path $l$ has no dramatic effect on $H_{\mathrm{c} 1}$ in the type-I regions, consistent with the fixed critical fields $\sim 130$ Oe from different PCS contacts.

For the type-II superconductor behavior among our PCS contacts, a critical field around 600 Oe is consistently observed without a large variation either. However, Fig. 5 indicates a slight change of the mean free path $l$ on surface should cause a wide distribution of $H_{\mathrm{c} 2}$ for $l \leq 200 \mathrm{~nm}$. Our observations probably argues against the mixture of type-I and type-II SC simply due to the disorder effect on surface. We notice that a variation of local density of states is frequently observed on the surface of several topological materials such as $\mathrm{Bi}_{2} \mathrm{Se}_{3}$, $\mathrm{Bi}_{2} \mathrm{Te}_{3}$ and $\mathrm{BiSbTeSe}{ }_{2}$, where charge puddles are proposed from a local fluctuation of the chemical potential when the Dirac cone is close to it [54-57]. The accumulated charge puddles on the surface should increase the local charge carriers substantially for the low-carrierdensity semimetal and thus reduce the mean free path $l$ with $l=\frac{3 \pi^{2} \hbar}{\rho e^{2} k_{F}^{2}}$ and $k_{F}=\sqrt[3]{3 \pi^{2} n}$. Once the carrier density $n$ goes beyond $4000 \times 10^{25} \mathrm{~m}^{-3}$, the reduced mean free path $l \sim 100 \mathrm{~nm}$ inside different puddles doesn't change too much as shown in the inset of Fig. 5, giving a critical field $H_{\mathrm{c} 2}$ between $400-700$ Oe. This crude model can roughly explain the disparate $H_{\mathrm{c}}$ values between type-I and type-II SC in our PCS results.

Our MPCS and SPCS spectra show a reproducible double peak structure with the absence of a zero-bias conductance peak and a perfect fitting with a single gap s-wave BTK model strongly supports a conventional swave paring gap in the type-II Dirac semimetal $\mathrm{PdTe}_{2}$ as evidenced by other measurements [30,34-38]. On the other hand, SC with a full gap is claimed for topological surface states to host Majorana zero modes in vortices for some iron-based superconductors [26-28]. As a typeI superconductor, it is thus difficult to check Majorana zero modes for $\mathrm{PdTe}_{2}$ without vortex lattice. However, a mixture of type-I and type-II SC on the surface offers a chance to search for Majorana fermions in $\mathrm{PdTe}_{2}$, if any. For $\mathrm{PdTe}_{2}$, this mixing is attributed to an inhomogeneous electron mean free path $l$ on the surface. More studies are needed to illuminate the relationship between this puzzling inhomogeneous behavior and topological surface states in $\mathrm{PdTe}_{2}$ especially by STM measurements.

In conclusion, we have observed a single full superconducting gap $\Delta$ from both MPCS and SPCS on the type II Dirac semimetal $\mathrm{PdTe}_{2}$ with $\Delta_{0} \sim 0.29 \mathrm{meV}$ and
$2 \Delta_{0} / k_{\mathrm{B}} T_{\mathrm{c}}=4.15$ in the strong coupling regime. However, the field-dependent conductance curves suggest a mixture of type-I and type-II SC with distinct critical fields probably due to an inhomogeneous electron mean free path on the surface of $\mathrm{PdTe}_{2}$. More careful studies are called for to address its origin and possible relations with topological surface states in $\mathrm{PdTe}_{2}$.

We are grateful for valuable discussions with L. Li, Y. Zheng and Y. Zhou. Our work was supported by the National Key Research \& Development Program of China (Grant No. 2016FYA0300402, 2017YFA0302901 and 2017YFA0303101) and the National Natural Science Foundation of China (Grant No. 11674279, 11774399 and 11374257). X.L. would like to acknowledge support from the Zhejiang Provincial Natural Science Foundation of China (LR18A04001) andY.G.S. acknowledges support from Beijing Natural Science Foundation (Z180008).

* Corresponding author: xinluphy@zju.edu.cn

[1] X. L. Qi and S. C. Zhang, "Topological insulators and superconductors," Rev. Mod. Phys. 83, 1057-1110 (2011).

[2] M. Z. Hasan and C. L. Kane, "Colloquium: Topological insulators," Rev. Mod. Phys. 82, 3045-3067 (2010).

[3] Y. Ando and L. Fu, "Topological crystalline insulators and topological superconductors: From concepts to materials," Annu. Rev. Condens. Matter Phys. 6, 361-381 (2015).

[4] M. Sato and Y. Ando, "Topological superconductors: a review," Rep. Prog. Phys. 80, 076501 (2017).

[5] N. Read and D. Green, "Paired states of fermions in two dimensions with breaking of parity and time-reversal symmetries and the fractional quantum Hall effect," Phys. Rev. B 61, 10267-10297 (2000).

[6] X. L. Qi, T. L. Hughes, S. Raghu, and S. C. Zhang, "Time-reversal-invariant topological superconductors and superfluids in two and three dimensions," Phys. Rev. Lett. 102, 187001 (2009).

[7] A. P. Schnyder, S. Ryu, A. Furusaki, and A. W. W. Ludwig, "Classification of topological insulators and superconductors in three spatial dimensions," Phys. Rev. B 78, 195125 (2008).

[8] A. Kitaev, "Periodic table for topological insulators and superconductors," AIP Conf. Proc. 1134, 22-30 (2009).

[9] C. Nayak, S. H. Simon, A. Stern, M. Freedman, and S. Das Sarma, "Non-Abelian anyons and topological quantum computation," Rev. Mod. Phys. 80, 1083-1159 (2008).

[10] A. Y. Kitaev, "Fault-tolerant quantum computation by anyons," Ann. Phys. 303, 2 - 30 (2003).

[11] C. W. J. Beenakker, "Search for Majorana fermions in superconductors," Annu. Rev. Condens. Matter Phys. 4, 113-136 (2013).

[12] M. Sato, "Topological properties of spin-triplet superconductors and Fermi surface topology in the normal state," Phys. Rev. B 79, 214526 (2009).

[13] L. Fu and E. Berg, "Odd-parity topological superconductors: Theory and application to $\mathrm{Cu}_{\mathrm{x}} \mathrm{Bi}_{2} \mathrm{Se}_{3}$," Phys. Rev. Lett. 105, 097001 (2010). 
[14] X. L. Qi, T. L. Hughes, and S. C. Zhang, "Topological invariants for the Fermi surface of a time-reversal-invariant superconductor," Phys. Rev. B 81, 134508 (2010).

[15] A. Yamakage, K. Yada, M. Sato, and Y. Tanaka, "Theory of tunneling conductance and surface-state transition in superconducting topological insulators," Phys. Rev. B 85, 180509 (2012).

[16] B. Lu, K. Yada, M. Sato, and Y. Tanaka, "Crossed surface flat bands of Weyl semimetal superconductors," Phys. Rev. Lett. 114, 096804 (2015).

[17] S. Kobayashi and M. Sato, "Topological superconductivity in Dirac semimetals," Phys. Rev. Lett. 115, 187001 (2015).

[18] G. Bednik, A. A. Zyuzin, and A. A. Burkov, "Superconductivity in Weyl metals," Phys. Rev. B 92, 035153 (2015).

[19] L. Fu and C. L. Kane, "Superconducting proximity effect and Majorana fermions at the surface of a topological insulator," Phys. Rev. Lett. 100, 096407 (2008).

[20] J. P. Xu, M. X. Wang, Z. L. Liu, J. F. Ge, X. Yang, C. H. Liu, Z. A. Xu, D. D. Guan, C. L. Gao, D. Qian, Y. Liu, Q. H. Wang, F. C. Zhang, Q. K. Xue, and J. F. Jia, "Experimental detection of a Majorana mode in the core of a magnetic vortex inside a topological insulator-superconductor $\mathrm{Bi}_{2} \mathrm{Te}_{3} / \mathrm{NbSe}_{2}$ heterostructure," Phys. Rev. Lett. 114, 017001 (2015).

[21] S. Y. Xu, N. Alidoust, I. Belopolski, A. Richardella, C. Liu, M. Neupane, G. Bian, S. H. Huang, R. Sankar, C. Fang, et al., "Momentum-space imaging of cooper pairing in a half-Dirac-gas topological superconductor," Nat. Phys. 10, 943 (2014).

[22] M. X. Wang, C. H. Liu, J. P. Xu, F. Yang, L. Miao, M. Y. Yao, C. L. Gao, C. Y. Shen, X. C. Ma, X. Chen, Z. A. Xu, Y. Liu, S. C. Zhang, D. Qian, J. F. Jia, and Q. K. Xue, "The coexistence of superconductivity and topological order in the $\mathrm{Bi}_{2} \mathrm{Se}_{3}$ thin films," Science 336, 52-55 (2012).

[23] J. P. Xu, C. H. Liu, M. X. Wang, J. F. Ge, Z. L. Liu, X. J. Yang, Y. Chen, Y. Liu, Z. A. Xu, C. L. Gao, D. Qian, F. C. Zhang, and J. F. Jia, "Artificial topological superconductor by the proximity effect," Phys. Rev. Lett. 112, 217001 (2014).

[24] P. Zareapour, A. Hayat, S. Y. Zhao, M. Kreshchuk, A. Jain, D. C. Kwok, N. Lee, S. W. Cheong, Z. J. Xu, A. Yang, et al., "Proximity-induced high-temperature superconductivity in the topological insulators $\mathrm{Bi}_{2} \mathrm{Se}_{3}$ and $\mathrm{Bi}_{2} \mathrm{Te}_{3}$," Nat. Commun. 3, 1056 (2012).

[25] E. Wang, H. Ding, A. V. Fedorov, W. Yao, Z. Li, Y. F. Lv, K. Zhao, L. G. Zhang, Z. J. Xu, J. Schneeloch, et al., "Fully gapped topological surface states in $\mathrm{Bi}_{2} \mathrm{Se}_{3} \overline{\text { films }}$ induced by a d-wave high-temperature superconductor," Nat. Phys. 9, 621 (2013).

[26] P. Zhang, K. Yaji, T. Hashimoto, Y. Ota, T. Kondo, K. Okazaki, Z. Wang, J. Wen, G. D. Gu, H. Ding, and S. Shin, "Observation of topological superconductivity on the surface of an iron-based superconductor," Science 360, 182-186 (2018).

[27] D. Wang, L. Kong, P. Fan, H. Chen, S. Zhu, W. Liu, L. Cao, Y.e Sun, S. Du, R. Schneeloch, J.and Zhong, G. Gu, L. Fu, H. Ding, and H. J. Gao, "Evidence for Majorana bound states in an iron-based superconductor," Science 362, 333-335 (2018).

[28] Q. Liu, C. Chen, T. Zhang, R. Peng, Y. J. Yan, C. H. P. Wen, X. Lou, Y. L. Huang, J. P. Tian, X. L. Dong, G. W.
Wang, W. C. Bao, Q. H. Wang, Z. P. Yin, Z. X. Zhao, and D. L. Feng, "Robust and clean Majorana zero mode in the vortex core of high-temperature superconductor (Li $\left.{ }_{0.84} \mathrm{Fe}_{0.16}\right)$ OHFeSe," Phys. Rev. X 8, 041056 (2018).

[29] H. J. Noh, J. Jeong, E. J. Cho, K. Kim, B. I. Min, and B. G. Park, "Experimental realization of type-II Dirac fermions in a $\mathrm{PdTe}_{2}$ superconductor," Phys. Rev. Lett. 119, 016401 (2017).

[30] O. J. Clark, M. J. Neat, K. Okawa, L. Bawden, I. Marković, F. Mazzola, J. Feng, V. Sunko, J. M. Riley, W. Meevasana, J. Fujii, I. Vobornik, T. K. Kim, M. Hoesch, T. Sasagawa, P. Wahl, M. S. Bahramy, and P. D. C. King, "Fermiology and superconductivity of topological surface states in $\mathrm{PdTe}_{2}$," Phys. Rev. Lett. 120, 156401 (2018).

[31] F. Fei, X. Bo, R. Wang, B. Wu, J. Jiang, D. Z. $\mathrm{Fu}, \mathrm{M}$. Gao, H. Zheng, Y. L. Chen, X. F. Wang, H. J. Bu, F. Q. Song, X. G. Wan, B. G. Wang, and G. Z. Wang, "Nontrivial Berry phase and typeII Dirac transport in the layered material $\mathrm{PdTe}_{2}$," Phys. Rev. B 96, 041201 (2017).

[32] W. Zheng, R. Schönemann, N. Aryal, Q. Zhou, D. Rhodes, Y.-C. Chiu, K.-W. Chen, E. Kampert, T. Förster, T. J. Martin, G. T. McCandless, J. Y. Chan, E. Manousakis, and L. Balicas, "Detailed study of the Fermi surfaces of the typeII Dirac semimetallic candidates $\mathrm{XTe}_{2}(\mathrm{X}=\mathrm{Pd}, \mathrm{Pt})$, Phys. Rev. B 97, 235154 (2018).

[33] H. Leng, C. Paulsen, Y. K. Huang, and A. de Visser, "Type-I superconductivity in the Dirac semimetal $\mathrm{PdTe}_{2}$," Phys. Rev. B 96, 220506 (2017).

[34] Amit and Y. Singh, "Heat capacity evidence for conventional superconductivity in the type-II Dirac semimetal $\mathrm{PdTe}_{2}$," Phys. Rev. B 97, 054515 (2018).

[35] S. Teknowijoyo, Na Hyun Jo, Mathias S. Scheurer, M. A. Tanatar, Kyuil Cho, S. L. Bud'ko, Peter P. Orth, P. C. Canfield, and R. Prozorov, "Nodeless superconductivity in the type-II Dirac semimetal $\mathrm{PdTe}_{2}$ : London penetration depth and pairing-symmetry analysis," Phys. Rev. B 98, 024508 (2018).

[36] M. V. Salis, P. Rodière, H. Leng, Y. K. Huang, and A. de Visser, "Penetration depth study of the type-I superconductor $\mathrm{PdTe}_{2}$," J.Phys.: Condens. Matter 30, 505602 (2018).

[37] A. Sirohi, S. Das, P. Adhikary, R. R. Chowdhury, A. Vashist, Y. Singh, S. Gayen, T. Das, and G. Sheet, "Mixed type I and type II superconductivity due to intrinsic electronic inhomogeneities in the type II Dirac semimetal $\mathrm{PdTe}_{2}$," J.Phys.: Condens. Matter 31, 085701 (2019).

[38] S. Das, Amit, A. Sirohi, L. Yadav, S. Gayen, Y. Singh, and G. Sheet, "Conventional superconductivity in the type-II Dirac semimetal $\mathrm{PdTe}_{2}$," Phys. Rev. B 97, 014523 (2018).

[39] G. E. Blonder, M. Tinkham, and T. M. Klapwijk, "Transition from metallic to tunneling regimes in superconducting microconstrictions: Excess current, charge imbalance, and supercurrent conversion," Phys. Rev. B 25, 4515-4532 (1982).

[40] D. Daghero and R. S. Gonnelli, "Probing multiband superconductivity by point-contact spectroscopy," Supercond. Sci. Technol. 23, 043001 (2010).

[41] S. Sasaki, M. Kriener, K. Segawa, K. Yada, Y. Tanaka, M. Sato, and Y. Ando, "Topological superconductivity 
in $\mathrm{Cu}_{\mathrm{x}} \mathrm{Bi}_{2} \mathrm{Se}_{3}$," Phys. Rev. Lett. 107, 217001 (2011).

[42] G. Sheet, S. Mukhopadhyay, and P. Raychaudhuri, "Role of critical current on the point-contact Andreev reflection spectra between a normal metal and a superconductor," Phys. Rev. B 69, 134507 (2004).

[43] Y. F. Lv, W. L. Wang, Y. M. Zhang, H. Ding, W. Li, L. L. Wang, K. He, C. L. Song, X. C. Ma, and Q. K. Xue, "Experimental signature of topological superconductivity and Majorana zero modes on $\beta-\mathrm{Bi}_{2} \mathrm{Pd}$ thin films," Sci. Bulletin 62, 852 - 856 (2017).

[44] T. Mizushima, A. Yamakage, M. Sato, and Y. Tanaka, "Dirac-fermion-induced parity mixing in superconducting topological insulators," Phys. Rev. B 90, 184516 (2014).

[45] J. Y. Guan, L. Y. Kong, L. Q. Zhou, Y. G. Zhong, H. Li, H. J. Liu, C. Y. Tang, D. Y. Yan, F. Z. Yang, Y. B. Huang, et al., "Experimental evidence of anomalously large superconducting gap on topological surface state of $\beta-\mathrm{Bi}_{2}$ Pd film," arXiv:1904.11638 (2019).

[46] G. E. Blonder and M. Tinkham, "Metallic to tunneling transition in $\mathrm{Cu}-\mathrm{Nb}$ point contacts," Phys. Rev. B 27, 112-118 (1983).

[47] Y. Huang, J. Yan, Y. L. Wang, L. Shan, Q. Luo, W. H. Wang, and H. H. Wen, "Isotropic swave pairing symmetry in non-centrosymmetric $\mathrm{Re}_{3} \mathrm{~W}$ revealed by point-contact spectroscopy," Supercond. Sci. Technol. 21, 075011 (2008).

[48] R. Prozorov, "Equilibrium topology of the intermediate state in type-I superconductors of different shapes," Phys. Rev. Lett. 98, 257001 (2007).

[49] V. Kozhevnikov, R. J. Wijngaarden, J. de Wit, and C. Van Haesendonck, "Magnetic flux density and the crit- ical field in the intermediate state of type-I superconductors," Phys. Rev. B 89, 100503 (2014).

[50] R. Prozorov, R. W. Giannetta, A. A. Polyanskii, and G. K. Perkins, "Topological hysteresis in the intermediate state of type-I superconductors," Phys. Rev. B 72, 212508 (2005).

[51] P. Parab, D. Singh, S. Haram, R. P. Singh, and S. Bose, "Point contact Andreev reflection studies of a non-centro symmetric superconductor $\mathrm{Re}_{6} \mathrm{Zr}$," Sci. Rep. 9, 2498 (2019).

[52] M. Tinkham, Introduction to superconductivity (Courier Corporation, 2004).

[53] A. E. Dunsworth, "The de Haas-van Alphen effect in $\mathrm{PdTe}_{2}$," J. Low Temp. Phys. 19, 51 (1975).

[54] T. Knispel, W. Jolie, N. Borgwardt, J. Lux, Z. Wang, Y. Ando, A. Rosch, T. Michely, and M. Grüninger, "Charge puddles in the bulk and on the surface of the topological insulator $\mathrm{BiSbTeSe}_{2}$ studied by scanning tunneling microscopy and optical spectroscopy," Phys. Rev. B 96, 195135 (2017).

[55] N. Borgwardt, J. Lux, I. Vergara, Zhiwei Wang, A. A. Taskin, Kouji Segawa, P. H. M. van Loosdrecht, Yoichi Ando, A. Rosch, and M. Grüninger, "Self-organized charge puddles in a three-dimensional topological material," Phys. Rev. B 93, 245149 (2016).

[56] Y. Zhang, V. W. Brar, C. Girit, A. Zettl, and M. F. Crommie, "Origin of spatial charge inhomogeneity in graphene," Nat. Phys. 5, 722 (2009).

[57] H. Beidenkopf, P. Roushan, J. Seo, L. Gorman, I. Drozdov, Y. San Hor, R. J. Cava, and A. Yazdani, "Spatial fluctuations of helical Dirac fermions on the surface of topological insulators," Nat. Phys. 7, 939 (2011). 